\title{
Feature Automata and Recognizable Sets of Feature Trees
}

\author{
Joachim Niehren ${ }^{\star}$ and Andreas Podelski \\ German Research Center for Artificial Intelligence (DFKI) \\ Stuhlsatzenhausweg 3, 6600 Saarbrücken 11 , Germany \\ niehren@dfki.uni-sb.de \\ Digital Equipment Corporation, Paris Research Laboratory (PRL) \\ 85, Avenue Victor Hugo, 92563 Rueil-Malmaison. France \\ podelskilprl.dec.com
}

\begin{abstract}
Feature trees generalize first-order trees whereby argument positions become keywords ("features") from an infinite symbol set $\mathcal{F}$. Constructor symbols can occur with any argument positions, in any finite number. Feature trees are used to model flexible records; the assumption on the infiniteness of $\mathcal{F}$ accounts for dynamic record field updates.

We develop a universal algebra framework for feature trees. We introduce the classical set-defining notions: automata, regular expressions and equational systems, and show that they coincide. This extension of the regular theory of trees requires new notions and proofs. Roughly, a feature automaton reads a feature tree in two directions: along its branches and along the fan-out of each node.

We illustrate the practical motivation of our regular theory of feature trees by pointing out an application on the programming language LIFE.
\end{abstract}

\section{Introduction}

In this section, we will give some background and motivation ("the task") and then oulline the rest of the paper ("the method").

The Task. We describe a specific formalism of data structures called feature trees. They are a generalization of first-order trees, also called constructor trees or the elements of the Herbrand universe. Since trees have been useful, e.g., for structuring data in modern symbolic programming languages like Prolog and ML, this gives the more flexible feature trees an interesting potential. Precisely, feature trees model record structures. They form the semantics of record calculi like [AK86], which are used in symbolic programming languages [AKP91b] and in computational linguistics ( $c f$., the book [Car92]). In the logical framcwork for record structures of [BS92], they constitute the interpretation of a completely axiomatizable, and hence decidable, first-order theory.

As graphs, feature trees are easily described as finite trees whose nodes are labeled by constructor symbols, and whose edges are labeled by feature symbols, all those edges outgoing from the same node by different oncs. Thus, symbolic keywords called features

\footnotetext{
^ partially supported by Graduierten-Kolleg Informatik der Universität des Saarlandes.
} 
denote the possible argument positions of a node. They access uniquely the node's direct subtrecs. All constructor symbols can label a node with any features attached to it, in any, though finite, number.

Although thoroughly investigated [AK86, Sm092, BS92, AKPS92], also in comparison with first-order trees [ST92], feature trees have never been characterized as composable elements in an algebraic structure, i.e., with operations defined on them. Also, up to now, there has been no corresponding notion of automata. This device has generally proven useful for dealing efficiently with systems calculating over sets of elements.

In our case, the practical motivation consists of the possibility of defining a hierarchy of types denoting sets of feature trees, as a Boolean lattice. For its use in a logical programming system employing feature trees such as LIFE [AKP91b], we need to compute efficiently the intersection of two types (roughly, for unification). Concurrent systems, in connection with control mechanisins such as residuation or guards [AKP91a], require furthermore an efficient test of the subset relation (matching). Thus, we need to provide a formalism defining the types in a way which is expressive enough and yet keeps the two problems decidable. Such a formalism can be given, for example, as a system of equations and a corresponding automata notion with Boolean closure properties and decidable emptyness problem.

A major difficulty of an algebraic framework for feature trees ${ }^{2}$ comes from the fact that the set $\mathcal{F}$ of features, i.e., of possible argument positions of a node accessing its direct subtrees, is infinite. The infiniteness of $\mathcal{F}$ is, however, an essential ingredient of the formal framcworks modeling flexible record structures. A practical motivation is the need to account for dynamic record field updates. It turns out that this semantical point of view has advantages in efficiency as well. Namely, the correctness of the algorithms for entailment and for solving negated constraints on feature trees [AKPS92] relies on the infiniteness of $\mathcal{F}$.

The Method. The first step in solving the problem described above is to build an appropriate algebraic framework. Such a framework is provided by universal algebra in the case of first-order trees. Formally, these are the elements of the free algebra over a given signature of function symbols (finite or infinite, $c f$., [Mah88]). This framework yields immediately a "good" notion of automata.

In fact, as Courcelle has shown in [Cou89, Cou92], universal algebra provides a framework for a rich variety of trees. Clearly, it is that work that inspired our notion of the algebra underlying fcature trees. We introduce this notion in Section 2. Informally speaking, the operation composing feature trees in the algebra takes a record value and adds a record field containing another value to it. In a special case, this amounts to Nivat's notion of 'sum of trees' [Niv92]; thus, incidentally, we obtain an algebraic formalization hereof.

To define feature automata as algebras, it is useful to consider the class of all finite trees whose nodes are labeled by constructor symbols, and whose edges are labeled by feature symbols. We call these multitrees. ${ }^{3}$ Multitrees are of interest on their own, namely for representation of knowledge with set-valued attributes [Rou88]. Thus, feature trees are

\footnotetext{
2 ... with the property that automata and equational systems coincide (let us note that the expressiveness of tree automata is equal to the one of equational systems for the free term algebras over finite signatures; it is strictly weaker in the case of infinite signatures for all tree species, also those considered in [Cou89, Cou92])

${ }^{3}$ The unranked unordered trees studied in [Cou89] (the number of arguments of the nodes is not
} 
multitrees with the restriction that features arc "functional," i.e., all edges outgoing from the same node are labeled with different fcatures. Feature automata recognize languages of multitrees, which are then cut down to recognize languages of feature trees.

In Section 3, we will define feature automata and show the basic properties of this notion: closure under the Boolean operations and decidability of the emptyness problem. In order to restrict our study to finitely presentable automata and yet to account for the infiniteness of the set of features $\mathcal{F}$, we introduce the notion of a finitary automaton: the number of states is finite, and the evaluation of the automaton can be specified not only on single symbols, but also on finite sets or on complements of finite sets of symbols. Thus, say: on $f$ for $f \in F$, or: on $f$ for $f \notin F$, where $F \subseteq \mathcal{F}$ finite.

Roughly, a feature automaton reads a feature tree in two directions: along its branches (from the frontier to the root) and along the fan-out of each node (along all argument positions). This is necessary in order to account for the flexibility in the depth as well as in the out-degree of the nodes of feature trees. The first direction is standard for all automata over trees. In order to study its behavior in the latter direction, or what we call the local structure of the recognized language, we consider recognizable sets of feature trees of depth 1 , called flat feature trees.

In Section 4, we define a class of logical formulas, called counting constraints. The name comes from the fact that they express threshold- or modulo counting of the subtrees which are accessed via features from a finite or co-finite set of features.

The main technical result of this paper is a theorem saying that counting constraints characterize exactly the recognizable sets of flat feature trees. The proof takes up Sections A and B. The theorem essentially links counting and the finitary-condition; in all of the set-defining devices presented here, either of these two notions accounts for the infiniteness of $\mathcal{F}$.

Counting constraints can express that certain features exist in the flat feature tree (labeling edges from the root), and that others do not. ${ }^{4}$ As a consequence, one can show that the set of first-order trees, with fixed arity assigned to constructor symbols, and recognizable subsets of these, are sets recognized by fcature automata.

In Sections 5 and 6 we give two alternative ways to define recognizable sets of feature trees which are more practical than automata: regular expressions and equational systems. In the first one, the sets are constructed by union, substitution and star (and, optionally, complement or intersection). In the second, they are defined as solutions of equations in a certain form. For both, counting constraints can be used to define the base cases. Thanks to the main theorem in Section 4, we are able to show that either class of defined sets is equal to the one for feature automata. Moreover, the devices can be effectively translated one into another. These results, logether with the previous ones, are necessary to present a complete regular theory of feature trees and to offer a solution to the practical problem of computing with types denoting sets of feature trees as described above.

restricted, and the arguments are not ordered) are a special case of multitrees, namely with just one feature. In the framework of [Cou89], however, recognizability by automata is strictly weaker than definability by equational systems, even if the set of node labels is finite.

${ }^{4}$ In [ST92, Smo92], these correspond to the constraints $x F, x f \downarrow$ or their negations, whete $F \subseteq \mathcal{F}$ finite and $f \in \mathcal{F}$. 


\section{The Algebra $\mathcal{J}$}

In this section we will introduce feature trees and the more general multitrees as elements of an algebra that we define, called $\mathcal{J}$. This yields the notion of a $\mathcal{J}$-automaton. This section follows the approach of [Cou89] and [Cou92].

In the following we will assume a given set $\mathcal{S}$ of constructor symbols (also called sorts, referred to by $A, B$, etc.) and a given set $\mathcal{F}$ of feature symbols (also called attributes, or record field selectors, referred to by $f, g$, etc.).

Formally, multitrees are trees (i.e., finite directed acyclic rooted graphs) whose nodes are labeled over $\mathcal{S}$, and whose edges are labeled over $\mathcal{F}$. Or, the set $\mathcal{M} \mathcal{T}$ of multitrees over $\mathcal{S}$ and $\mathcal{F}$ can be introduced as $\mathcal{M T}=\bigcup_{n \geq 0} \mathcal{M} \mathcal{T}_{n}$ where (let $\mathbf{N}$ denote the set of all natural numbers, and $\mathbb{N}_{\text {finite }}^{M}$ the set of finite multisets with elements from the set $M$ ):

$$
\begin{aligned}
& \mathcal{M} \mathcal{T}_{0}=\{(A, \emptyset) \mid A \in \mathcal{S}\} \\
& \mathcal{M} \mathcal{T}_{n}=\left\{(A, E) \mid A \in \mathcal{S}, E \in \mathbb{N}_{\text {finite }}^{\left.\mathcal{F} \times \mathcal{M} \mathcal{T}_{n-1}\right\} \cup \mathcal{M} \mathcal{T}_{n-1} .}\right.
\end{aligned}
$$

$\mathcal{M} \mathcal{T}_{n}$ contains the multitrees of depth $\leq n$.

Feature trees are multitrees such that all edges outgoing from the same node are labeled by different features. $\mathcal{F T}$ denote the set of all feature trees (and $\mathcal{F} \mathcal{T}_{n}$ all those of depth $\leq n$ ).

We introduce two sorts $M T$ and $F$ for multitrees and features, respectively, and define the $\{M T, F\}$-sorted signature:

$$
\Sigma=\{\Rightarrow\} \uplus \mathcal{F} \uplus \mathcal{S}
$$

where $\Rightarrow$ is a function symbol of profile: $M T \times F \times M T \mapsto M T$, and the symbols in $\mathcal{F}$ and $S$ are constants of sort $F$ and of sort $M T$, respectively.

The algebra of multitrees $\mathcal{J}$ is defined as a $\Sigma$-algebra. Its two domains are $D_{M T}=\mathcal{M T}$ and $D_{F}=\mathcal{F}$ of the sorts $M T$ and $F$, respectively. The function symbol $\Rightarrow$ is interpreted in $\mathcal{J}$ as the operation which composes two multitrees $t, t^{\prime} \in \mathcal{M T}$ via a feature $f \in \mathcal{F}$ to a new multitree composed of $t$ and $t^{\prime}$ with an edge labeled $f$ from the root of $t$ to the root of $t^{\prime}$. Or (where $\cup$ denotes multiset union),

$$
\Rightarrow^{\mathcal{J}}((A, E), f, t)=(A, E \sqcup\{(f, t)\}) .
$$

Borrowing the 'tree sum' notation from [Niv92], we might write $\Rightarrow^{\mathcal{J}}\left(t, f, t^{\prime}\right)$ more intuitively as $t+f t^{\prime}$. In fact, for the special case where $\mathcal{F}=\{1,2\}$ (the two features denoting left and right successors), we obtain an algebraic reading of the notation of [Niv92].

The interpretation of the constants is given by $f^{\mathcal{J}}=f$ and $A^{\mathcal{J}}=(A, \emptyset)$.

It is easy to verify that the algebra $\mathcal{J}$ satisfies the order independence (OIT), i.e., the following equation is valid in $\mathcal{J}$.

$$
\Rightarrow\left(\Rightarrow\left(x, f_{1}, x_{1}\right), f_{2}, x_{2}\right)=\Rightarrow\left(\Rightarrow\left(x, f_{2}, x_{2}\right), f_{1}, x_{1}\right)
$$

In the 'tree sum' notation this expresses the commutativity ${ }^{5}$ of + , in the sense that $t+f_{1} t_{1}+f_{2} t_{2}=t+f_{2} t_{2}+f_{1} t_{1}$. Of course, always $t+f_{1} t_{1}+f_{2} t_{2} \neq t+f_{1}\left(t_{1}+f_{2} t_{2}\right)$.

We use $\mathcal{T}_{\Sigma}$ to denote the free algebra of terms over the signature $\Sigma$.

\footnotetext{
${ }^{5}$ In a sense which can be made formal ( $c f$., Section A), also the associativily holds for + ; this justifies dropping the parenthesis.
} 
Lemma 1. The algebra of multitrees $\mathcal{J}$ is isomorphic to the quotient of the free term algebra over $\Sigma$ with the least congruence generated by the order-independence equation(1),

$$
\mathcal{J}=\mathcal{T}_{\boldsymbol{\Sigma} / O I T}
$$

It is well-known that, given any system of equations $\mathcal{E}, \mathcal{T}_{\Sigma / \mathcal{E}}$ is the initial object in the category of all $\Sigma$-algebras satisfying the equations $\mathcal{E}$.

A $\mathcal{J}$-automaton is a tuple $\left(\mathcal{A}, h, Q_{\text {final }}\right)$ consisting of a finite $\Sigma$-algebra $\mathcal{A}$, a homomorphism $h: \mathcal{J} \mapsto \mathcal{A}$ and the subsel $Q_{\text {final }} \subseteq D_{M T}^{\mathcal{A}}$ of values of sort $M T$ ("final states") where the number of values of sort $M T$ and of sort $F$ ("states") is finite. A $\mathcal{J}$-automaton corresponds to the "more concrete" notion of a (finite deterministic bottom-up) tree automaton over the terms of $T_{\Sigma}$ such that all terms which are equal modulo OIT are evaluated to the same state. This means that any representation of a multitree $t$ as a term in $T_{\Sigma}$ can be chosen in order to calculate the value of $t$.

\section{Feature Automata}

Given any many-sorted signature $\Sigma$ with a finite number of non-constant function symbols $c \in) \Sigma-\Sigma_{s}^{0}$ for every sort $s$, we define a $\Sigma$-algebra $\mathcal{A}$ to be finitary if, for each sort $s$ and each value $q \in D_{s}^{\mathcal{A}}$ of sort $\mathrm{s}$, the set:

$$
\left\{c \in \Sigma_{s}^{0} \mid c^{\mathcal{A}}=q\right\}
$$

of constant symbols in $\Sigma$ of sort s which are valued to $q$ is finite or co-finite.

We now return to the particular $\{M T, F\}$-sortcd signature $\Sigma$ introduced above; clearly, the definitions bclow can be made in general framework as wcll. ${ }^{6}$

A feature automaton $\mathcal{A}$ is defined as a finitary $\mathcal{J}$-automaton. The set of multitrees recognized by $\mathcal{A}$ is the set:

$$
L_{\mathcal{M} T}(\mathcal{A})=\left\{t \in \mathcal{M T} \mid h(t) \in Q_{\text {final }}\right\},
$$

and the set of feature trees recognized by $\mathcal{A}$ is the set: $L_{\mathcal{F} \mathcal{T}}(\mathcal{A})=L_{\mathcal{M} T}(\mathcal{A}) \cap \mathcal{F T}$. The families $\operatorname{Rec}_{\mathcal{M} \tau}(\mathcal{J})$ and $\operatorname{Rec} \mathcal{F} \tau(\mathcal{J})$ of recognizable sets of multitrees and feature trees are defined accordingly.

Remark. If (and only if) the set of features is infinite, the set of all feature trees is not a recognizable language of multitrecs (with respect to $\mathcal{J}$ ).

Example. We will construct a feature automaton $\mathcal{A}$ that recognizes the set of natural numbers. These are coded into the feature trecs of the form $(0,\{(\operatorname{succ},(0,\{(\operatorname{succ},(\ldots,\{(0, \emptyset)\})\})\})\})$, with $n$ edges labeled succ for the natural number $n$. As clements in the quotient term algebra $\mathcal{T}_{\Sigma / \mathrm{OIT}}$, they would be written as the singleton congruence classes $\{\Rightarrow(0, s u c c, \Rightarrow$

${ }^{6}$ Also, the finitary-condition: finite or co-finite, could be made more general such that the proof of Theorem 2 still holds. 
$(0$, succ, $\Rightarrow(\ldots, 0)))\}$. The feature automaton $\mathcal{A}$ has the states $Q=\left\{q_{\text {nat }}, q_{\text {other }}\right\}$ and $P=\left\{p_{\text {succ }}, p_{\text {other }}\right\}$ of sort $M T$ and $F$, respectively. The evaluation is given by:

$$
\begin{aligned}
& 0^{\mathcal{A}}=q_{\text {nat }}, \\
& A^{\mathcal{A}}=q_{\text {other }} \quad \text { if } A \neq 0 \text {, } \\
& \operatorname{succ}^{\mathcal{A}}=p_{\text {succ }} \text {, } \\
& f^{\mathcal{A}}=p_{\text {other }} \text { if } f \neq s u c c, \\
& \Rightarrow^{\mathcal{A}}\left(q_{\text {nat }}, p_{\text {succ }}, q_{\text {nat }}\right)=q_{\text {nat }}, \\
& \Rightarrow^{\mathcal{A}}\left(q_{1}, p, q_{2}\right)=q_{\text {other }} \text { otherwise. }
\end{aligned}
$$

As final state set we choose $Q_{\text {final }}=\left\{q_{n a t}\right\}$. It is clear that $\mathcal{A}$ respects the order independence theory and the finitary-condition. Of course, it will be more practical to define this set by regular expressions or equational systems.

The following theorem and corollary states that the standard properties of recognizable languages are valid for the sets in $\operatorname{ReCF}_{\mathcal{F} T}$ as well.

\section{Theorem 2.}

1. The family of recognizable languages of feature trees RecFT is closed under the Boolean operations. The corresponding feature automata can be given effectively.

2. The emptiness problem $\left(L_{\mathcal{F} T}(\mathcal{A}) \stackrel{?}{=} \emptyset\right)$ is decidable for each feature automaton $\mathcal{A}$.

Proof. The known constructions for Boolean operations on automata are still valid for $\mathcal{J}$-automata. To see that the finitary-condition is preserved, simply note that the system of finite and co-finite sets is Boolean closed and, for two states $q_{1}$ and $q_{2}$ of the feature automata $\mathcal{A}_{1}$ and $\mathcal{A}_{2}$, respectively,

$$
\left\{c \in \Sigma_{s}^{0} \mid c^{\left(\mathcal{A}_{1}, \mathcal{A}_{2}\right)}=\left(q_{1}, q_{2}\right)\right\}=\left\{c \in \Sigma_{s}^{0} \mid c^{\mathcal{A}_{1}}=q_{1}\right\} \cap\left\{c \in \Sigma_{s}^{0} \mid c^{\mathcal{A}_{2}}=q_{2}\right\} .
$$

Since $\mathcal{J}=\mathcal{T}_{\Sigma / \mathrm{OIT}}$, cach $\mathcal{J}$-automaton $\mathcal{A}$ corresponds to a tree automaton $\mathcal{A}_{T}$ over terms in $T_{\Sigma}$, and:

$$
L_{\mathcal{F} T}(\mathcal{A})=\emptyset \text { iff } L_{T_{\Sigma}}\left(\mathcal{A}_{T}\right)=\emptyset,
$$

it suffices to decide the emptiness problem for the tree automaton $\mathcal{A}_{T}$. As usually, this can be done by checking all terms of depth smaller than the number of states of $\mathcal{A}_{T}$. Let $C$ be some finite set of constants $c$ such that $c^{\mathcal{A}}=q$ for each state $q$ which is a value of some constant. Iff $L$ is not empty, it contains a term of bounded depth that is constructed with constants of $C$ and non-constant function symbols. But there are only finitely many terms of this kind.

A finitary automaton can be finitely represented. From such a representation onc can calculate some set $C$ as described above. This yields an algorithm for testing $L_{\mathcal{M} \tau}(\mathcal{A})=\emptyset$. In the case of $L_{\mathcal{F} T}(\mathcal{A})$ the algorithm checks only terms representing feature trees.

We conclude the section by defining non-deterministic feature automata which are needed in Sections 5 and 6. 
Definition 3. A non-deterministic feature automaton $\mathcal{A}=\left(Q, P, h, Q_{\text {final }}\right)$ is a tuple such that:

$Q$ is the set of states of sort $M T, P$ the states of sort $F$ and $Q_{\text {final }} \subseteq Q$ is the set of final states, $h$ is composed of the functions $h: S \rightarrow 2^{Q}$ and $h: \mathcal{F} \rightarrow 2^{P}$ and the transition function $\Rightarrow^{\mathcal{A}}: Q \times P \times Q \rightarrow 2^{Q}$,

$\mathcal{A}$ satisfies the OIT-theory, i.e., for all states $q, p_{1}, q_{1}, p_{2}, q_{2}$,

$$
\Rightarrow^{\mathcal{A}}\left(\Rightarrow^{\mathcal{A}}\left(q, p_{1}, q_{1}\right), p_{2}, q_{2}\right)=\Rightarrow^{\mathcal{A}}\left(\Rightarrow^{\mathcal{A}}\left(q, p_{2}, q_{2}\right), p_{1}, q_{1}\right)
$$

$\mathcal{A}$ satisfies the finitary-condition, i.e., for all states $p$ and $q$, the sets $\left\{f \in \mathcal{F} \mid p \in f^{\mathcal{A}}\right\}$ and $\left\{A \in \mathcal{S} \mid q \in A^{\mathcal{A}}\right\}$ are finite or co-finite.

The evaluation of the term $t \in \mathcal{T}_{\Sigma}$ by $\mathcal{A}$, i.e., the $\operatorname{set} h(t) \subseteq Q$ is defined inductively by:

$$
h\left(\Rightarrow\left(t_{1}, f, t_{2}\right)\right)=\Rightarrow^{\mathcal{A}}\left(h\left(t_{1}\right), h(f), h\left(t_{2}\right)\right)
$$

If $t_{1}$ and $t_{2}$ are congruent modulo OIT, we have $h\left(t_{1}\right)=h\left(t_{2}\right)$. Thus, $h([t])=h(t)$ is well defined for all congruence classes $[t]$. The language of multitrees recognized by $\mathcal{A}$ is:

$$
L_{\mathcal{M} T}(\mathcal{A})=\left\{[t] \mid h([t]) \cap Q_{\text {final }} \neq \emptyset\right\}
$$

and the language of feature trees recognized by $\mathcal{A}$ is $L_{\mathcal{F} T}(\mathcal{A})=L_{\mathcal{M} \tau}(\mathcal{A}) \cap \mathcal{F T}$. Each feature automaton is also a non-deterministic feature automaton.

Lemma 4. Given a non-deterministic feature automaton $\mathcal{A}$, an equivalent (deterministic) feature automaton $\mathcal{A}^{d}$ can be constructed effectively.

Proof We apply the usual subset construction on a given non-deterministic feature automaton $\mathcal{A}$ of the form above, yielding the equivalent automaton $\mathcal{A}^{d}$ as follows: $Q^{d}=2^{Q}, P^{d}=2^{P}, A^{\mathcal{A}^{d}}=A^{\mathcal{A}}, f^{\mathcal{A}^{d}}:=f^{\mathcal{A}}$, and:

$$
\Rightarrow^{\mathcal{A}^{d}}\left(q_{1}^{d}, p^{d}, q_{2}^{d}\right)=\bigcup\left\{\Rightarrow^{\mathcal{A}}\left(q_{1}, p, q_{2}\right) \mid\left(q_{1}, p, q_{2}\right) \in q_{1}^{d} \times p^{d} \times q_{2}^{d}\right\}
$$

We define the final states of $\mathcal{A}^{d}$ by: $Q_{\text {final }}^{d}=\left\{q^{d} \mid q^{d} \cap Q_{\text {final }} \neq \emptyset\right\}$.

Clearly, the algebra $\mathcal{A}^{d}$ satisfies the $O I T$-theory. The equality: The finitary-condition is preserved, since:

$$
\left\{A \mid A^{\mathcal{A}^{d}}=q^{d}\right\}=\bigcap_{q \in q^{d}}\left\{A \mid q \in A^{\mathcal{A}}\right\} \cap \bigcap_{q \notin q^{d}}\left\{A \mid q \in A^{\mathcal{A}}\right\}^{C}
$$

shows that the finitary-condition is prescrved, too. 


\section{Counting Constraints}

In this section we characterize recognizable languages of flat feature trees using formulae of a certain from, called counting constraints. The proof of this characterization, which is the main technical result of this paper, will be done in Sections A and B.

The syntax of counting constraints $C$ (writlen $C(x)$ to indicate that $x$ is the only free variable) is defined in the BNF style as follows (where $F$ a finite or co-finite sets of features, $n, m \in \mathbb{N}$ are natural numbers, and $T$ is a finite or co-finite subset of $\mathcal{S}$ ).

$$
\begin{aligned}
C(x)::= & \operatorname{card}\{\varphi \in F \mid \exists y \cdot(x \varphi y \wedge T y)\}=n \bmod m \\
& \mid \begin{array}{l}
S x \\
C(x) \wedge C(x) \\
\neg C(x)
\end{array}
\end{aligned}
$$

The counting constraint $C(x) \equiv \operatorname{card}\{\varphi \in F \mid \exists y .(x \varphi y \wedge T y)\}=n \bmod m$ holds for the multitree $x$ if the number of all edges in $x$ which: (1) go from the root to a node labeled by a symbol in $T$ and (2) are labeled by a feature $\varphi$ in $F$, is equal to $n \bmod m .^{7}$ The cardinality operator card applies on a multiset of features, i.e., counts their double occurrences.

The counting constraint $C(x) \equiv S x$ holds for the multitree $x$ if the root of $x$ is labeled by some symbol in $S$.

We note the following fact (cf., [Eil74]).

Fact 5 A language of natural numbers is recognizable iff it can be decomposed into a finite union of sets of the form: $\{n+k \cdot m \mid k \in \mathbb{N}\}$, with $n, m \in \mathbb{N}$.

Thus, we can define the syntax of counting constraints equivalently in the form (where $N$ is a set of natural numbers which is recognizable in the monoid $(\mathbb{N},+, 0) ; S$, and $T$, a finite or co-finite subset of $\mathcal{S} ; F$ a finite or co-finite sets of features):

$$
\begin{aligned}
& C(x)::= \operatorname{card}\{\varphi \in F \mid \exists y .(x \varphi y \wedge T y)\} \in N \\
& \mid S x \\
& \mid C(x) \wedge C(x) \\
& C(x) \vee C(x)
\end{aligned}
$$

Note that also under this definition, counting constraints are closed under negation. Indeed, $\neg \operatorname{card}\{\varphi \in F \mid \exists y .(x \varphi y \wedge T y)\} \in N$ is equivalent to $\operatorname{card}\{\varphi \in F \mid \exists y .(x \varphi y \wedge T y)\} \in N^{c}$, and $\neg T x$ is equivalent to $T^{c} x$.

Some important feature constraints can be expressed by our new constraints. For example, in the syntax of [Smo92], for $F \subseteq \mathcal{F}$ finite, for $f \in \mathcal{F}$, and for $A \in \mathcal{S}: x F$ ("for cxactly the features $f$ in $F$ there exists one edge labeled $f$ from the root"), $x f \downarrow$ ("there exists no edge labcled $f$ from the root"), and $A x$ ("the root is labeled by $A$ ").

$$
x F \equiv \bigwedge_{f \in F} \operatorname{card}\{\varphi \in\{f\} \mid \exists y \cdot x \varphi y\} \in\{1\}
$$

\footnotetext{
${ }^{7}$ We define $n \bmod 0=n$, although this is not quite standard. That is, "counting" means here thresholdand modulo counting.
} 


$$
\begin{aligned}
\wedge \quad \operatorname{card}\left\{\varphi \in F^{c} \mid \exists y . x \varphi y\right\} \in\{0\}, \\
x f \downarrow \equiv \operatorname{card}\{\varphi \in\{f\} \mid \exists y . x \varphi y\} \in\{0\}, \\
A x \equiv\{A\} x .
\end{aligned}
$$

Each constraint $C(x)$ defines the set $L_{M T}(C)$ of multitrees $x$ for which the constraint $C(x)$ holds. Accordingly, we define: $L_{F T}(C)=L_{M T}(C) \cap \mathcal{F T}, L_{M T_{1}}(C)=L_{M T}(C) \cap \mathcal{M} \mathcal{T}_{1}$, and $L_{F T_{1}}(C)=L_{F T}(C) \cap \mathcal{F} \mathcal{T}_{1}$. The languages of flat multitrees of the form $L_{M T_{1}}(C)$, or of nat feature trees $L_{F T_{1}}(C)$, are called counting-definable.

The following theorem holds for multitrees instead of feature trees, as well.

Theorem 6. A language of flat feature trees is counting-definable iff it is recognizable (in $\mathcal{J}$, by a feature automaton).

Proof Sketch. A flat multitree can be represented as a finite multiset over $(\mathcal{F} \cup\{$ rool $\}) \times \mathcal{S}$. The operation $\Rightarrow \mathcal{J}$ corresponds to the union of such multisets. In Section A we study the algebra $\mathcal{M}$ of finite multisets of pairs. It is three-sorted, the sorts denoting $\mathcal{F} \cup\{$ root $\}, \mathcal{S}$ and $\mathcal{M} \mathcal{T}$, respectively. We show that $\mathcal{J}$ - and $\mathcal{M}$-recognizability coincide.

In Section $B$, we consider counting constraints $D(x)$ for multisets $x$ of $\mathcal{M}$. They are of the form:

$$
D(x) \equiv \operatorname{card}\{(f, A) \in x \mid f \in F, A \in T\} \in N,
$$

or conjunctions or disjunctions of these. Again $F$ and $T$ are finite or co-finite subsets of $\mathcal{F}$ and $S$ and $N$ is a recognizable set of natural numbers.

We show that definability of languages of multisets by these constraints and $\mathcal{M}$ recognizability coincide. The main idea is that the mapping:

$$
x \mapsto \operatorname{card}\{(f, A) \in x \mid f \in F, A \in T\}
$$

is essentially a homomorphism from $\mathcal{M}$ into $\mathbb{N}$.

The theorem above expresses that feature automata can count features either threshold or modulo a natural number.

\section{Kleene's Theorem}

We define regular expressions over feature trees. In generalization of the standard cases, the atomic constituents of these are not just constants (denoting singletons or trees of depth 1), but expressions which denote sets of feature trees of depth $\leq 1$.

As usual, we need construction variables labeling the nodes where the substitution and the Kleene star operations can take place. These variables are taken from a set $Y$ which is assumed given (disjoint from $\mathcal{S}$ ). It is infinite; the definition of each regular language, of course, uses only a finite number of construction variables. We call a syntactic expression $C$ of the form (2) a counting-expression if $T$ ranges over finite or co-finite subsets of $\mathcal{S} \cup Y$. 
Its denotation is defined as the set of all feature trees of depth $\leq 1$ which satisfy it as a counting constraint over the extended alphabet of sorts.

A regular expression $R$ over $\mathcal{F}$ and $\mathcal{S} \cup Y$ is of the form given by:

$$
R::=\begin{array}{ll}
C & C \text { is a counting-expression } \\
\mid R \cdot y, R & \text { concatenation (where } y \in Y \text { ) } \\
\mid R^{\star_{y}} & \text { Kleene star (where } y \in Y \text { ) } \\
\mid R \cup R & \text { union }
\end{array}
$$

Complement and intersection are optional operators, which, as we will see, do not properly add expressiveness.

The definition of the language $L_{\mathcal{F} T}(R)$ of feature trees (or $L_{\mathcal{M T}}(R)$ of multitrees) denoted by the regular expression $R$ is by straightforward induction. For concatenation and Kleene star for sets of multitrees: If $L_{1}$ and $L_{2}$ are sets of feature trees, then $L_{1} \cdot{ }_{y} L_{2}$ is obtained by replacing the construction variable $y$ in the leaves of the trees of $L_{1}$ by (possibly different) trees of $L_{2}$. The Kleene star operation on a set is an iterated concatenation of a set with itself. Formally, for a set. $L$ of feature trees, $L_{y}^{1}=L, L_{y}^{n}:=L_{y}^{n-1}{ }_{y} L$, and $L^{\star_{y}}=\bigcup_{n \geq 1} L_{y}^{n}$. The languages of feature trees (or multitrees) denoted by regular expressions are called regular languages.

Theorem 7 Kleene. A language of feature trees (or multitrees) is regular iff it is recognizable.

Proof. It is sufficient to prove the theorem for multitrees. We show by induction over the structure of the regular expressions that the language of each regular expression over $\mathcal{S} \cup Y$ and $\mathcal{F}$ is recognizable. The base case $R=C$ is handled by Theorem 6 . Union is captured by the Boolean closure properties in Theorem 2. Substitution and star are established using the equivalence of deterministic and non deterministic feature automata. For the other direction, we use the standard McNaughton/Papert induction technique, the base case being handled again by Theorem 6 .

\section{Equational Systems}

The next possibility to define recognizable sets of feature trees (or multitrees) in a convenient way uses equational systems. These systems again generalize the constituents from singletons of trees of the form $a$ or $f\left(y_{1}, \ldots, y_{n}\right)$, for $a \in \Sigma_{0}$ and $f \in \Sigma_{n}$ in the case of a ranked signature for first-order trees, to counting-expressions denoting (unions of) sets of flat feature trees.

The extra symbols $y \in Y$ in these counting expressions now correspond to set variables of the equations.

We write $C\left(y_{1}, \ldots, y_{n}\right)$ instead of $C$ if the set variables of $C$ are contained in the set $\left\{y_{1}, \ldots, y_{n}\right\}$. These variables are not to be confused with the logical variable $x$ used in $C=C(x)$ as a logical formula.

An equational system is a finite set $\mathcal{E}$ of equations of the form (where $C_{i}$ is a countingexpression, for $i=1, \ldots, n)$ :

$$
y_{i}=C_{i}\left(y_{1}, \ldots, y_{n}\right) \text {. }
$$


Given an assignment, i.e., a mapping $\alpha: Y \mapsto 2^{\mathcal{F} \mathcal{T}}$, the cquations in $\mathcal{E}$ are interpreted such that $C_{i}\left(y_{1}, \ldots, y_{n}\right)$ denotes the set:

$$
L_{\mathcal{F} T}\left(C_{i}\right) \cdot_{y_{1}} \alpha\left(y_{1}\right) \cdot_{y_{2}} \ldots y_{n} \alpha\left(y_{n}\right) .
$$

A solution of $\mathcal{E}$ is an assignment $\alpha$ satisfying $\mathcal{E}$. Each equational system has a least solution. The existence follows with the usual fixed point argument. Namely, an equational system is considered as an operator over the lattice of assignments $\alpha$ and the least solution is obtained in $\omega$ itcration steps of this operator, starting with the assignment $\alpha\left(y_{i}\right)=\emptyset$ for $i=1, \ldots, n$.

A language of feature trees is called equational if it is the union of some of the sets $\alpha\left(y_{i}\right)$ for the lcast solution $\alpha$ of $\mathcal{E}$. The notion is defined accordingly for multitrees.

Theorem 8. A language of fealure trees (or multitrees) is equational iff it is recognizable.

Proof Since $\mathcal{J}$-recognizability corresponds to the characterization by congruence relations, and Theorem 6 covers the case of feature trees of depth $\leq 1$, the proof can be done following the standard one for first-order trees ( $c f_{.},[$GS84]).

\section{Conclusion and Further Work}

The results of this paper together present a complete regular theory of feature trees. They offer a solution to the concrete practical problem of computing with types denoting sets of feature trees as described in the introduction.

Now, it is interesting to investigate where, in the wide range of applications of first-order trees, feature trees can be useful in replacing or extending those. Since tree automata play a major role, either directly or just by underlying some other formalism, the regular theory of feature trees developed here is a prerequisite for this investigation.

A more speculative application might be conceived as part of the compiler optimizer of the programming language LIFE [AKP91b]. Namely, unary predicates over feature trees defined by Horn clauses without multiple occurrences of variables define recognizable sets of feature trees. Now, satisfiability of the conjunction of two such predicates corresponds to non-emptyness of the intersection of the defined sets. When used in deep guards, entailment of a predicate by others of this kind corresponds to the subset relation on the defined sets of feature trees.

We are curious to extend the developed theory in the following ways. First, we would like to find a logical characterization of the class of recognizable feature trees, cxtending the results of Doner, Thatcher/Wright and Courcelle [Don70, TW67, Cou90]. It will be interesting to combine second-order logic and the counting constraints introduced here, in order to account for the flexibility in the depth as well as in the out-degree of the nodes of feature trees.

Also, in order to account for circular data structures, like, e.g., circular lists, it is necessary to consider infinite (rational) feature trees. Thus, it would be useful to construct a regular theory of these.

Finally, in [CD91] it is shown that the first-order theory of a tree automaton is decidable (in the case of a finite signature). More precisely, it is possible to solve first-order formulas 
built up from equalities between first-order terms and membership constraints of the form $x \in q$, where $q$ denotes a set defined by a tree automaton. Since we have established the corresponding automaton notion, we may hope to obtain the corresponding result for feature trees. For the special case of the set of all feature trees, this is the decidability of first-order feature logic. A proof for infinite feature trees can be found in [BS92]. Can the techniques of that proof be combined with the ones of [CD91]?

We add the fact, suggested by one of the referees, that the first-order theory of multitrees is not decidable. This can be shown by employing a proof technique by Ralf Treinen [Trei92].

\section{Acknowledgements}

We are grateful to Hassan Aït-Kaci and Gert Smolka for first arousing our individual interest in the idea of using tree automata for feature constraint solving and then bringing us together. We also thank Hubert Comon, Helmut Seidl and Ralf Treinen for encouraging discussions. Finally, we thank the anonymous referees for their helpful and intriguing comments.

\section{References}

[AK86] Hassan Ait-Kaci. An algebraic semantics approach to the effective resolution of type equations. Theoretical Computer Science, 45:293-351, 1986.

[AKP91a] Hassan Aït-Kaci and Andreas Podelski. Functional constraints in LIFE. PRL Research Report 13, Digital Equipment Corporation, Paris Research Laboratory, Rueil-Malmaison, France, 1991.

[AKP91b] Hassan Aïl-Kaci and Andreas Podelski. Towards a meaning of LIFE. In J. Maluszyníski and M. Wirsing, editors, Proceedings of the 3rd International Symposiumon Programining Language Implementation and Logic Programming, Springer LNCS vol. 528, pages 255274. Springer-Verlag, 1991.

[AKPS92] Hassan Aïl-Kaci, Andreas Podelski, and Gert Smolka. A feature-based constraint system for logic programming with entailment. In Proceedings of the 5th International Conference on Fifih Generation Computer Systems, pages 1012-1022, Tokyo, Japan, June 1992. ICOT.

[BS92] Rolf Backofen and Gert Smolka. A complete and recursive feature theory. Research Report RR-92-30, German Research Center for Artificial Intelligence (DFKI), Stuhlsatzenhausweg 3, 6600 Saarbrücken 11, Germany, September 1992.

[Car92] Bob Carpenter. The Logic of Typed Fealure Structures, volume 32 of Cambridge Tracts in Theorelical Computer Science. Cambridge University Press, Cambridge, UK, 1992.

[CD91] Hubert Comon and Catherine Delors. Equational formula with membership constraints,. Rapport de recherche 648, LRI, Universit de Paris Sud, March 1991. To appear in Information and Computation.

[Cou89] Bruno Courcelle. On recognizable sets and tree automata. In Hassan Ait-Kaci and Maurice Nivat, editors, Resolution of Equations in Algebraic Structures, Algebraic Techniques, volume 1, chapter 3, pages 93-126. Academic Press, 1989.

[Cou90] Bruno Courcelle. The monadic second-order logic of graphs I: Recognizable sets of finite graphs. Information and Compulation 85, pages 12-75, 1990.

[Cou92] Bruno Courcelle. Recognizable sets of unrooted trees. In Maurice Nivat and Andreas Podelski, editors, Tree Aulomata, Advances and Open Problems. Elsevier Science, 1992.

[Don70] John E. Doner. Tree Acceptors and some of their applications. Journal of Comp. System Sci. 4, pages 406-451, 1970. 
[Ei174] Samuel Eilenberg. Automata, Language and Machine, volume A of Applied and Pure Mathematics. Academic Press, 1974.

[GS84] F. Gécseg and M. Steinby. Tree Automata. Akadémiai Kiadó, Budapest, 1984.

[Mah88] Michael J. Maher. Complete axiomatizations of the algebras of finite, rational and infinite trees. In LICS, pages 348-457, July 1988.

[Niv92] Maurice Nivat. Elements of a theory of tree codes. In Maurice Nivat and Andreas Podelski, editors, Tree Automala, Advances and Open Problems. Elsevier Science, 1992.

[Rou88] William C. Rounds. Set values for unification-based grammar formalisms and logic programming. Report CSLI-88-129, 1988.

[Sm092] Gert Smolka. Feature constraint logics for unification grammars. Journal of Logic Programming, 12:51-87, 1992.

[ST92] Gert Smolka and Ralf Treinen. Records for logic programming. In Proceedings of the 1992 Joint International Conference and Symposium on Logic Programming, Washington, DC, November 1992. The MIT Press, to appear.

[TW67] J. W. Thatcher and J. B. Wright. Generalized finite automata theory with an application to a decision problem of second-order logic. Mathematical Systems Theory, 2(1):57-81, August 1967. Published by Springer-Verlag NY Inc.

[Trei92] Ralf Treinen. A New Method for Undecidability Proofs of First Order Theories. Journal of Symbolic Computation, 14:437-457, 1992.

\section{APPENDIX}

\section{A The Algebra of Multisets}

We will reduce $\mathcal{J}$-recognizability for languages of flat multitrees to a notion of recognizability of finite multisets of pairs. The idea is to identify a flat multitree with a finite multiset of pairs,

$$
(A, E) \equiv\{(\operatorname{rool}, A)\} \sqcup E
$$

where root is considered like an extra feature. Roughly, the operation of adding edges corresponds to the union operation on multisets.

In all generality, we introduce the algebra $\mathcal{M}=\mathcal{M}\left(\mathcal{U}_{1}, \ldots, \mathcal{U}_{n}\right)$ of finite multisets over $n$-luples with components in given sets $\mathcal{U}_{1}, \ldots, \mathcal{U}_{n}$, for some $n \geq 1$. (Later, we will instantiate $\mathcal{U}_{1}=\mathcal{F} \cup\{$ root $\}$ and $\mathcal{U}_{2}=\mathcal{S}$.) $\mathcal{M}$ is $n+1$-sorted, over the the sorts $s_{1}, \ldots, s_{n}$ and $F M S$ which denote, respectively, the domains $D_{s_{1}}=\mathcal{U}_{1}, \ldots, D_{s_{n}}=\mathcal{U}_{n}$, and $D_{F M S}=\mathbb{N}_{\text {finite }} \mathcal{U}_{1} \times \ldots \times \mathcal{U}_{n}$ (where $\mathbb{N}_{\text {finite }}^{M}$ denotes the set of finite multisets over $M$ ).

The operations of $\mathcal{M}$ are the (associative and commutative) union $\omega^{\mathcal{M}}$ of multisets and the creation of a singleton multiset from $n$ elcments, one for each component, i.e., $\left\langle u_{1}, \ldots, u_{n}\right\rangle^{\mathcal{M}}=\left\{\left(u_{1}, \ldots, u_{n}\right)\right\}$. Thus, they are mappings $\sqcup^{\mathcal{M}}: D_{F M S} \times D_{F M S} \mapsto D_{F M S}$, and \langle\rangle$^{\mathcal{M}}: \mathcal{U}_{1} \times \ldots \times \mathcal{U}_{n} \mapsto D_{F M S}$.

Formally, $\mathcal{M}$ is an algebra over the $\left\{s_{1}, \ldots, s_{n}, F M S\right\}$-sorted signature:

$$
\Sigma_{u_{1}, \ldots, \mathcal{U}_{n}}=\mathcal{U}_{1} \uplus \ldots \uplus \mathcal{U}_{n} \uplus\{\langle., \ldots, .\rangle, \sqcup\}
$$

where the constants of sort $s_{i}$ are just the elements of $\mathcal{U}_{i}$, and the two function symbols have the profile: $\downarrow: F M S \times F M S \mapsto F M S$, and \langle\rangle$: s_{1} \times \ldots \times s_{n} \mapsto F M S$. 
Lemma9. The algebra $\mathcal{M}$ is isomorphic to the quotient of the term algebra with the congruence generated by the associativity and commutativity laws for $\sqcup$,

$$
\mathcal{M}=T_{\Sigma_{u_{1}, \ldots, u_{n} / A C}}
$$

We define a subset of $D_{F M T}$ of multisets of $n$-tuples to be recognizable if it is recognized by a finitary $\mathcal{M}$-automaton.

It is important to note that the notions of recognizability in $\mathcal{M}=\mathcal{M}\left(\mathcal{U}_{1}, \ldots, \mathcal{U}_{n}\right)$ and $\mathcal{M}\left(\mathcal{U}_{1} \times \ldots \times \mathcal{U}_{n}\right)$ can be different, namely if $n \geq 2$ and one of the $\mathcal{U}_{i}$ is infinite.

Now, we consider the special case where $\mathcal{U}_{1}=\mathcal{F} \cup\{$ root $\}$ and $\mathcal{U}_{2}=\mathcal{S}$, i.e.,

$$
\mathcal{M}=\mathcal{M}(\mathcal{F} \cup\{\text { root }\}, \mathcal{S}) \text {. }
$$

Thus, the domains of $\mathcal{M}$ are $D_{s_{1}}^{\mathcal{M}}=\mathcal{F} \cup\{$ root $\}, D_{s_{2}}^{\mathcal{M}}=\mathcal{S}$, and $D_{F M S}^{\mathcal{M}}=F M S(\mathcal{F} \cup$ $\{$ root $\} \times \mathcal{S})$.

We define the injection:

$$
I: \mathcal{M} \mathcal{T}_{1} \rightarrow \mathbb{N}_{\text {finite }}^{(\mathcal{F} \cup\{\text { root }\}) \times \mathcal{S}}
$$

by $I((A, E))=\{($ root,$A)\} \cup E$. Thus (writing the operator $\sqcup^{\mathcal{M}}$ infix):

$$
I\left(\Rightarrow^{\mathcal{J}}(t, f, A)\right)=I(t) \sqcup^{\mathcal{M}}\langle f, A\rangle^{\mathcal{M}}
$$

Lemma 10 Reduction Lemma. A language L of flat multitrees is recognizable in $\mathcal{J}$ iff the language $I(L)$ of multisets of pairs is recognizable in $\mathcal{M}$.

Proof The difficult direction is from left to right. Given a finitary $\mathcal{J}$-automaton $\left(\mathcal{A}, h, Q_{\text {tinal }}\right)$, where $D_{\mathcal{A}}^{M T}=Q$ and $D_{\mathcal{A}}^{F}=P$, we construct a finitary $\mathcal{M}$-automaton $\left(\mathcal{A}^{\star}, h^{\star}, Q_{\text {final }}\right)$ such that, for all flat multitrees $t$ :

$$
h^{\star}(I(t))=h(t)
$$

This is sufficient to show the recognizability of $I(L)$, since $I(L)=h^{-1}(\mathcal{A}) \cap I\left(\mathcal{M T _ { 1 }}\right)$, and $I\left(\mathcal{M} T_{1}\right)$ is a recognizable set in $\mathcal{M}$.

We set $D_{s_{2}}^{\mathcal{A}^{*}}=Q, D_{s_{1}}^{\mathcal{A}^{*}}=P \cup\left\{p_{\text {root }}\right\}$, and (wherc Func denotes the set of functions generated by the functions $\Rightarrow^{\mathcal{T}}(., p, q)$; i.e., the smallest set containing these and closed under composition):

$$
D_{F M S}^{\mathcal{A}^{\star}}=\text { Func } \uplus Q \uplus\left\{q_{\perp}\right\} \text {. }
$$

\footnotetext{
${ }^{8}$ Generally, the finiteness condition for $\mathcal{M}\left(\mathcal{U}_{1} \times \ldots \times \mathcal{U}_{n}\right)$-automata is weaker then the one for $\mathcal{M}$-automata. It may be strictly weaker since cartesian products of finite and co-finite sets need neither be finite nor co-finite. For example, suppose $\mathcal{U}$ to be an infinite set. The cartesian product $\mathcal{U} \times\{1\}$ is neither finite nor co-finite as subset of $\mathcal{U} \times\{0,1\}$. Thus, the language of the singleton subsets of $\mathcal{U} \times\{1\}$ is not recognizable in the algebra $\mathcal{M}(\mathcal{U} \times\{0,1\})$, but it is with respect to $\mathcal{M}=\mathcal{M}(\mathcal{U},\{0,1\})$. - In fact, it is this finitary-condition which makes the proofs that complicated and non-standard.
} 
The evaluation of $\mathcal{A}^{\star}$ is defined by (we write $\cdot \mathcal{A}^{\star}$ instead of $h^{\star}(\cdot)$ and use the more intuitive infix notation):

$$
\begin{aligned}
\langle p, q\rangle^{\mathcal{A}^{*}} & =\Rightarrow^{\mathcal{A}}(., p, q), \\
\left\langle p_{\text {root }}, q\right\rangle^{\mathcal{A}^{*}} & =q, \\
h_{1} \cup^{\mathcal{A}^{*}} h_{2} & =h_{1} \circ h_{2}, \\
q \cup^{\mathcal{A}^{*}} h & =h(q), \\
h \cup^{\mathcal{A}^{*}} q & =h(q), \\
q \sqcup^{\mathcal{A}^{*}} \tilde{q} & =q_{\perp} .
\end{aligned}
$$

Every function in the interpretations taking $q_{\perp}$ as argument is again mapped to $q_{\perp}$. Precisely:

$$
\begin{aligned}
q_{\perp} \cup^{\mathcal{A}^{*}} h & =q_{\perp}, \\
h \sqcup^{\mathcal{A}^{*}} q_{\perp} & =q_{\perp}, \\
q_{\perp} \sqcup^{\mathcal{A}^{*}} q & =q_{\perp}, \\
q \cup^{\mathcal{A}^{*}} q_{\perp} & =q_{\perp}, \\
\left\langle p, q_{\perp}\right\rangle^{\mathcal{A}^{*}} & =q_{\perp}, \\
\left\langle p_{\text {rool }}, q_{\perp}\right\rangle^{\mathcal{A}^{*}} & =q_{\perp} .
\end{aligned}
$$

Clearly, $\mathcal{A}^{\star}$ is an $A C$-automaton,i.e., the operation $\sqcup^{\mathcal{A}^{\star}}$ is associative and commutative. The associativity is trivial for functions as arguments. The commutativity for functions follows from the OIT -theory, and the associativity for functions by:

$$
\begin{aligned}
\Rightarrow^{\mathcal{A}}(., p, q) \sqcup^{\mathcal{A}^{*}} \Rightarrow^{\mathcal{A}}\left(., p_{1}, q_{1}\right) & \left.=\Rightarrow^{\mathcal{A}}\left(\Rightarrow^{\mathcal{A}}\left(., p_{1}, q_{1}\right), p, q\right)\right) \\
& \left.=\Rightarrow^{\mathcal{A}}\left(\Rightarrow^{\mathcal{A}}(., p, q), p_{1}, q_{1}\right)\right) \\
& =\Rightarrow^{\mathcal{A}}\left(., p_{1}, q_{1}\right){\sqcup^{\mathcal{A}}}^{*} \Rightarrow^{\mathcal{A}}(., p, q) .
\end{aligned}
$$

The proof for all possible cases is now easily established.

The identity (4) is now easily verified. Finally, we note that the finitary-condition is preserved from $\mathcal{A}$ to $\mathcal{A}^{*}$.

For the other direction, given a finitary $\mathcal{M}$-automaton $\mathcal{A}^{\star}$, we will construct a finitary $\mathcal{J}$-automaton $\mathcal{A}$ satisfying (4). This is sufficient, now, since $\mathcal{M} \mathcal{T}_{1}$ is a recognizable set in $\mathcal{J}$. In fact, we will construct an automaton in another algebra. ${ }^{9} \mathrm{Next}$, we will introduce this algebra. We resume this proof after having proven Lemma 12.

The algebra $\mathcal{J}_{\text {local }}$ of flat multitrees is obtained from the algebra $\mathcal{J}$ by restricting the domain of the third argument from $\mathcal{M T}$ to $S\left(\ldots=\mathcal{M} \mathcal{T}_{0}\right)$, and the domain of the first from $\mathcal{M T}$ to $\mathcal{M} \mathcal{T}_{1}$, i.e., to to flat multitrees instead of arbitrary ones.

That is, the algebra $\mathcal{J}_{\text {local }}$ is three-sorted with sorts $M T_{1}, F$ and $S$. The domains are given by $D_{M T_{1}}=\mathcal{M} \mathcal{T}_{1}, D_{F}=\mathcal{F}, D_{S}=\mathcal{S}$. The operation is given by (where $E$ is a finite multiset over pairs in $\mathcal{F} \times \mathcal{S}$ ):

$$
\Rightarrow^{\mathcal{J}_{\text {iocal }}}\left(\left(A_{1}, E\right), f, A_{2}\right)=\left(A_{1}, E \sqcup\left\{\left(f, A_{2}\right)\right\}\right)
$$

(which is equal to $\Rightarrow^{\mathcal{J}}\left(\left(A_{1}, E\right), f, A_{2}\right)$ ). The signature of $\mathcal{J}_{\text {local }}$ is the disjoint union:

$$
\Sigma_{\text {local }}=\mathcal{S} \uplus \mathcal{F} \uplus \mathcal{S} \uplus\{\Rightarrow\} \text {. }
$$

\footnotetext{
${ }^{9}$ The motivation for the construction of yet another algebra is, roughly, the fact that a symbol $A \in \mathcal{S}$ occurs as a root-labeling as well as a leave-labeling; these two roles are distinguished in $\mathcal{J}$-automata, but not in $\mathcal{M}$-automata.
} 
Here, the symbols in $\mathcal{S}$ appear twice: they are supposed to be renamed apart. Firstly, they are constants of sort $M T_{1}$, and secondly, they are constants of sort $S$. The different functionality is made clear syntactically by writing $A_{M T_{1}}$ and $A_{S}$, with interpretations $\left(A_{M T_{1}}\right)^{J_{\text {locat }}}=(A, \emptyset) \in \mathcal{M} \mathcal{T}_{0} \subseteq \mathcal{M} \mathcal{T}_{1}$ and $\left(A_{S}\right)^{J_{\text {local }}}=A \in \mathcal{S}$.

The features are constants of sort $F$ and interpreted freely. The profile of the function symbol in $\mathcal{J}_{\text {local }}$ is $\Rightarrow: M T_{1} \times F \times S \rightarrow M T_{1}$.

The algebra $\mathcal{J}_{\text {local }}$ satisfies the order independence theory (OIT); namely, for all flat multitrees $t$, features $\int$ and symbols $A$ the following holds.

$$
\Rightarrow \mathcal{J}^{\mathcal{J}_{\text {local }}}\left(\left(\Leftrightarrow \mathcal{J}_{\text {locat }}\left(t, f_{1}, A_{1}\right), f_{2}, A_{2}\right)=\Rightarrow^{\mathcal{J}_{\text {local }}}\left(\left(\Rightarrow \mathcal{J}_{\text {local }}\left(t, f_{2}, A_{2}\right), f_{1}, A_{1}\right)\right.\right.
$$

The following lemma states that we can use the more concrete notion of tree automata.

Lemma 11. $\mathcal{J}_{\text {local }}$ is isomorphic to a quotient term algebra,

$$
\mathcal{J}_{\text {local }}=\mathcal{T}_{\Sigma_{\text {loosd }} / \text { OIT }}
$$

Again, we define recognizability in $\mathcal{J}_{\text {local }}$ in terms of finitary automata.

Lemma 12. A language of flat multitrees is recognizable in $\mathcal{J}$ iff it is recognizable in $\mathcal{J}_{\text {local. }}$

Proof We will first modify a finitary $\mathcal{J}$-automaton $\mathcal{A}$, where $D_{\mathcal{A}}^{M T}=Q$ and $D_{\mathcal{A}}^{F}=P$, in order to obtain a finitary $\mathcal{J}_{\text {local }}$-automaton $\mathcal{A}^{1}$ such that the two automata (with the same set of final states) will recognize the same languages of flat multitrees. We define the domains of $\mathcal{A}^{1}$ by:

$$
\begin{aligned}
D_{S}^{\mathcal{A}^{1}} & =Q, \\
D_{M T_{1}}^{\mathcal{A}^{1}} & =Q, \\
D_{F}^{\mathcal{A}^{1}} & =P,
\end{aligned}
$$

and we define the evaluation of $\mathcal{A}^{1}$ by (for all $A \in \mathcal{S}, f \in \mathcal{F}$, and for all $q, q^{\prime} \in Q$ and $p \in P)$ :

$$
\begin{aligned}
\left(A_{M T_{1}}\right)^{\mathcal{A}^{1}} & =A^{\mathcal{A}}, \\
\left(A_{S}\right)^{\mathcal{A}^{1}} & =A^{\mathcal{A}}, \\
f^{\mathcal{A}^{1}} & =f^{\mathcal{A}}, \\
\Rightarrow^{\mathcal{A}^{1}}\left(q, p, q^{\prime}\right) & =\Rightarrow^{\mathcal{A}}\left(q, p, q^{\prime}\right) .
\end{aligned}
$$

Clearly the finitary-condition and the order independence theory are preserved between $\mathcal{A}^{1}$ and $\mathcal{A}$.

For the other direction, given a finitary $\mathcal{J}_{\text {local }}$-automaton $\mathcal{A}^{2}$ (with final states $Q_{\text {final }}^{2}$, of sort $M T_{1}$ ), we will define a finitary $\mathcal{J}_{\text {local }}$-automaton $\mathcal{A}^{1}$ that recognizes the same language, but has the two properties: $D_{M T_{1}}^{\mathcal{A}^{1}}=D_{S}^{\mathcal{A}^{1}}$, and, for all symbols $A$ in $\mathcal{S},\left(A_{M T_{1}}\right)^{\mathcal{A}^{1}}=\left(A_{S}\right)^{\mathcal{A}^{1}}$. Thanks to these, one can define a $\mathcal{J}$-automaton $\mathcal{A}$ that accepts the same flat multitrces as $\mathcal{A}^{1}$. Again, this is sufficient since the language $\mathcal{M} \mathcal{T}_{1}$ is recognizable with respect to $\mathcal{J}$. 
We define the domains of $\mathcal{A}^{1}$ by:

$$
\begin{aligned}
D_{M T_{1}}^{\mathcal{A}^{1}} & =D_{M T_{1}}^{\mathcal{A}^{2}} \times D_{S}^{\mathcal{A}^{2}}, \\
D_{S}^{\mathcal{A}^{1}} & =D_{M T_{1}}^{\mathcal{A}^{2}} \times D_{S}^{\mathcal{A}^{2}}, \\
D_{F}^{\mathcal{A}^{1}} & =D_{F}^{\mathcal{A}^{2}},
\end{aligned}
$$

and, after having fixed an arbitrary element $r_{f i x} \in D_{S}^{\mathcal{A}^{2}}$, we define the evaluation of $\mathcal{A}^{1}$ by (for all $A \in \mathcal{S}, f \in \mathcal{F}$, and for all $q, \tilde{q} \in D_{\mathscr{M}_{1}}^{\mathcal{A}_{1}^{2}}, p \in D_{F}^{\mathcal{A}^{2}}$ and $r, \tilde{r} \in D_{\mathcal{S}}^{\mathcal{A}^{2}}$ ):

$$
\begin{aligned}
\left(A_{M T_{1}}\right)^{\mathcal{A}^{1}} & =\left(\left(A_{M T_{1}}\right)^{\mathcal{A}^{2}},\left(A_{S}\right)^{\mathcal{A}^{2}}\right), \\
\left(A_{S}\right)^{\mathcal{A}^{1}} & =\left(\left(A_{M T_{1}}\right)^{\mathcal{A}^{2}},\left(A_{S}\right)^{\mathcal{A}^{2}}\right), \\
f^{\mathcal{A}^{1}} & =f^{\mathcal{A}^{2}}, \\
\Rightarrow \mathcal{A}^{1}((q, r), p,(\tilde{q}, \tilde{r})) & =\left(\Rightarrow \mathcal{A}^{2}(q, p, \tilde{r}), r_{f i x}\right) .
\end{aligned}
$$

As final states of $\mathcal{A}^{1}$ we choose:

$$
Q_{\text {final }}^{1}=\left\{(q, r) \mid q \in Q_{\text {final }}^{2} \text { and } r \in D_{S}^{\mathcal{A}^{2}}\right\} .
$$

Again, the finiteness condition and the order independence theory are preserved. This concludes the proof of Lemma 12.

\section{End of Proof of Reduction Lemma 10}

Given a finitary $\mathcal{M}$-automaton $\mathcal{A}^{\star}$, we construct a finitary $\mathcal{J}_{\text {local }}$-automaton $\mathcal{A}$ such that $(I(t))^{\mathcal{A}^{\star}}=\mathcal{A}^{\mathcal{A}}$ for all flat multitrees $t$. The domains of $\mathcal{A}$ are: $D_{S}^{\mathcal{A}}=D_{s_{2}}^{\mathcal{A}^{\star}}, D_{F}^{\mathcal{A}}=D_{s_{1}}^{\mathcal{A}^{\star}}$ and $D_{M T_{1}}^{\mathcal{A}}=D_{F M S}^{\mathcal{A}^{\star}}$.

The evaluation of $\mathcal{A}$ is defined by (where $q, p$ and $r$ are states of $\mathcal{A}$ of sorts $M T_{1}, F$ and $S$ ):

$$
\begin{aligned}
\left(A_{S}\right)^{\mathcal{A}} & =A^{\mathcal{A}^{*}}, \\
f^{\mathcal{A}} & =f^{\mathcal{A}^{*}}, \\
\left(A_{M T_{1}}\right)^{\mathcal{A}} & =\left\langle\operatorname{root}^{\mathcal{A}^{*}},\left(A_{S}\right)^{\mathcal{A}^{*}}\right\rangle^{\mathcal{A}^{*}}, \\
\Rightarrow^{\mathcal{A}}(q, p, r) & =q \omega^{\mathcal{A}^{*}}\langle p, r\rangle^{\mathcal{A}^{*}} .
\end{aligned}
$$

Since $\mathcal{A}^{\star}$ satisfies (AC), $\mathcal{A}$ satisfies (OIT). The finitary-condition is preserved, as well.

\section{B Counting in Multisets}

Again in the general framework where $\mathcal{M}=\mathcal{M}\left(\mathcal{U}_{1}, \ldots, \mathcal{U}_{n}\right)$, We will characterize recognizability in $\mathcal{M}$, i.e., of languages of finitc multiscts, by appropiate counting constraints.

We define $\mathcal{M}$-counting constraints $C$ (written $C(x)$ to indicate that $x$ is the only free variable-logically, a multiset variable) to expressions of the following form:

$$
\begin{aligned}
C(x)::= & \operatorname{card}\left\{\left(u_{1}, \ldots, u_{n}\right) \in x \mid u_{i} \in U_{i} \text { for all } i\right\} \in N \\
& \mid C(x) \cap C(x) \\
& \mid C(x) \cup C(x) .
\end{aligned}
$$


Here, $N$ is a recognizable set of natural numbers with respect to the monoid $(\mathbb{N},+, 0)$, and the sets $U_{i} \subset \mathcal{U}_{i}$ are finite or co-finite. The counting constraint

$C(x) \equiv \operatorname{card}\left\{\left(u_{1}, \ldots, u_{n}\right) \in x \mid u_{i} \in U_{i}\right.$ for all $\left.i\right\} \in N$ holds for the multiset $x$ if the number of tuples $\left(u_{1}, \ldots, u_{n}\right)$ in $x$ such that $u_{i} \in U_{i}$ for all $i=1, \ldots, n$ is an element of $N$. The cardinality operater card applies on a multiset of tuples, $i . e$. , counts double occurrences.

The language defined by an $\mathcal{M}$-counting constraint $C(x)$ is the set of all finite multisets $x$ that satisfy $C(x)$. It is denoted by $L_{\mathcal{M}}(C)$.

Theorem 13. The family of languages defined by $\mathcal{M}$-counting constraints is exactly the family of languages of multisets recognizable in $\mathcal{M}$.

Proof. Given an $\mathcal{M}$-counting constraint of the form: $C=\operatorname{card}\left\{\left(u_{1}, \ldots, u_{n}\right) \in x \mid u_{i} \in\right.$ $U_{i}$ for all $\left.i\right\} \in N$, we will show the recognizability of $L_{\mathcal{M}}(C)$.

We can define a homomorphism $h: \mathcal{M}\left(\mathcal{U}_{1} \ldots, \mathcal{U}_{n}\right) \rightarrow \mathcal{M}(\{1\}, \ldots,\{1\})$ by setting $h\left(u_{i}\right)=\{1\}$ for $u_{i} \in U_{i}$, and $h\left(u_{i}\right)=\emptyset$ otherwise.

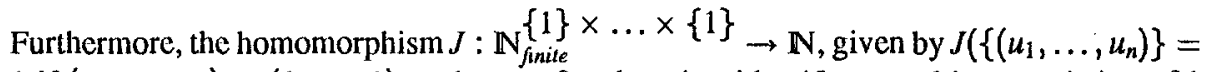
1 if $\left(u_{1}, \ldots, u_{n}\right)=(1, \ldots, 1)$, and $\ldots=0$, otherwise, identifies a multiset consisting of $k$ tuples $(1, \ldots, 1)$ with $k \in \mathbb{N}$.

Thus, for all finite multisets of $n$-tuples $x \in D_{F M T}$,

$$
J(h(x))=\operatorname{card}\left\{\left(u_{1}, \ldots, u_{n}\right) \in x \mid u_{i} \in U_{i} \text { for all } i\right\} .
$$

Hence, $L_{\mathcal{M}}(C)=h^{-1}\left(J^{-1}(N)\right)$. The finitary-condition is invariant under inverse images of homomorphisms. Thus, $L_{\mathcal{M}}(C)$ is recognizable in $\mathcal{M}$.

For the reverse inclusion, suppose that $L$ is recognized by a finitary $\mathcal{M}$-automaton $\left(\mathcal{A}, h, Q_{\text {final }}\right)$ with, say, the set $D_{F M S}=\left\{q_{1}, \ldots, q_{n}\right\}$ of states of sort FMS.

The evaluation of the multiset $t$ by $\mathcal{A}$ leads to the state (written in a notation which is justified by the fact that $\mathcal{A}$ satisfies (AC), even if $\cup^{\mathcal{A}}$ is taken over the empty multiset):

$$
t^{\mathcal{A}}=\bigsqcup_{\left(u_{1}, \ldots, u_{n}\right) \in t}^{\mathcal{A}}\left\langle u_{1}^{\mathcal{A}}, \ldots, u_{n}^{\mathcal{A}}\right\rangle^{\mathcal{A}}
$$

We define the natural numbers: $a_{t}(i)=\operatorname{card}\left\{\left(u_{1}, \ldots, u_{n}\right) \in t \mid\left\langle u_{1}^{\mathcal{A}}, \ldots, u_{n}^{\mathcal{A}}\right\rangle^{\mathcal{A}}=q_{i}\right\}$ and obtain (again thanks to (AC) bcing satisfied):

$$
t^{\mathcal{A}}=\bigsqcup_{i=1}^{n} \mathcal{A} \bigsqcup_{j=1}^{a_{r}(i)} \mathcal{A} q_{i}
$$

We define a mapping $\nu_{t}:\{1, \ldots, n\} \rightarrow\{1, \ldots, n\}$ such that $q_{\nu_{t}(i)}=\bigsqcup_{j=1}^{a_{1}(i) \mathcal{A}} q_{i}$. If $t \in L_{\mathcal{M}}(\mathcal{A})$, then:

$$
\bigsqcup_{i=1}^{n} \mathcal{A} q_{\nu_{t}(i)} \in Q_{\text {finai }}
$$


Generally, for a mapping $\mu:\{1, \ldots, n\} \rightarrow\{1, \ldots, n\}$, we define, for $i=1, \ldots, n$, the set of natural numbers:

$$
N_{\mu}^{i}=\left\{m \in \mathbb{N} \mid \bigsqcup_{j=1}^{m}{ }^{\mathcal{A}} q_{i}=q_{\mu(i)}\right\} .
$$

We note that $a_{i}(i) \in N_{\nu_{1}}^{i}$ for $i=1, \ldots, n$. That is, $t$ is an element of the language defined by the $\mathcal{M}$-counting constraint:

$$
\bigwedge_{i=1}^{n} a_{x}(i) \in N_{\nu_{i}}^{i}
$$

Vice versa, for each mapping $\mu$ satisfying the property (5), the language of the $\mathcal{M}$-counting constraint:

$$
\bigwedge_{i=1}^{n} a_{x}(i) \in N_{\mu}^{i}
$$

is contained in $L$. We get $L=L(R)$ where $R$ is the $\mathcal{M}$-counting constraint:

$$
R=\bigvee_{\substack{\mu \\ \text { with (s) }}} \bigwedge_{i=1}^{n} a_{x}(i) \in N_{\mu}^{i}
$$

Since the number of mappings $\mu$ with (5) is finite, it only remains to show that the constraints used in $R$ are of the defined form. The constituents $a_{i}(x)$ are admissible by the finitary-condition of $\mathcal{A}$. Finally, we have to proof that the sets $N_{\mu}^{i}$ are recognizable with respect to $(\mathbb{N},+, 0)$. We will construct appropiate automata $\mathcal{A}_{\mu}^{i}$ from $\mathcal{A}$. We set $D^{\mathcal{A}_{\mu}^{i}}=Q$, $0^{\mathcal{A}_{\mu}^{i}}=\emptyset^{\mathcal{A}}, 1^{\mathcal{A}_{\mu}^{i}}=q_{i}$ and interpret the addition by $\sqcup^{\mathcal{A}}$. As final states we take the singleton $\left\{q_{\mu(i)}\right\}$. Then, $\mathcal{A}_{\mu}^{i}$ recognizes $N_{\mu}^{i}$.

\section{Proof of Theorem 6.}

For each language $L$ of flat multitrees defined by a counting constraint $C$ we will find an $\mathcal{M}$-counting constraint $C^{\prime}$ that defines $I(L)$, and vice versa.

Given a counting constraint for flat multitrees of the form:

$$
C(x)=\operatorname{card}\{\varphi \in F \mid \exists y \cdot(x \varphi y \wedge T y\} \in N,
$$

we sct:

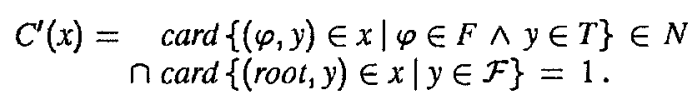

The case $C=T x$ is obvious, as well as conjunction and disjunction.

For the other direction, given an $\mathcal{M}$-counting constraint $C^{\prime}$ for finite multisets, we will give a constraint $C$ such that $L_{\mathcal{M} T_{1}}\left(C_{x}\right)=I^{-1}\left(L_{\mathcal{M}}\left(C^{\prime}\right)\right)$, or, equivalently, $L_{\mathcal{M} T_{1}}(C)=$ $L_{\mathcal{M}}\left(C^{\prime}\right) \cap I\left(\mathcal{M} T_{1}\right)$. We note that the languages of the form $I(L)$ are the multisets containing exactly one pair with first component root. Given the $\mathcal{M}$-counting constraint:

$$
C^{\prime}=\operatorname{card}\{(\varphi, y) \in x \mid \varphi \in F \wedge y \in T\} \in N
$$


we have to distinguish the two cases root $\notin F$ and root $\in F$. In the first case we set:

$$
C=\operatorname{card}\{\varphi \in F \mid \exists y .(x \varphi y \wedge T y\} \in N .
$$

In the second case, we note that the set: $N-1=\{n-1 \mid n \in N$ and $n \geq 1\}$ is recognizable with respect to $(\mathcal{N},+, 0)$, and set:

$$
C=\underset{\cap T x .}{\operatorname{card}}\{\varphi \in F-\{\text { root }\} \mid \exists y .(x \varphi y \wedge T y)\} \in N-1
$$

In either case $C$ has the required property.

This concludes the proof of Theorem 6, since the reduction lemma (Lemma 10, page 14) and the above theorem (Theorem 13) close the cycle from counting-definable languages $L$ of flat feature trees to those recognizable in $\mathcal{J}$ by feature automata. Namely, according to the above correspondence between counting- and $\mathcal{M}$-counting constraints, via $\mathcal{M}$-counting-definable languages $I(L)$, which, according to Theorem 13 , ate exactly the ones recognizable in $\mathcal{M}$, back to $L$ according to Lemma 10. 